\title{
IMPROVED LINEAR DISCRIMINANT ANALYSIS CONSIDERING EMPIRICAL PAIRWISE CLASSIFICATION ERROR RATES
}

\author{
Hung-Shin Lee and Berlin Chen \\ Department of Computer Science and Information Engineering, \\ National Taiwan Normal University, Taipei
}

\begin{abstract}
Linear discriminant analysis (LDA) is designed to seek a linear transformation that projects a data set into a lower-dimensional feature space for maximum class geometrical separability. LDA cannot always guarantee better classification accuracy, since its formulation is not in light of the properties of the classifiers, such as the automatic speech recognizer (ASR). In this paper, the relationship between the empirical classification error rates and the Mahalanobis distances of the respective class pairs of speech features is investigated, and based on this, a novel reformulation of the LDA criterion, distance-error coupled LDA (DE-LDA), is proposed. One notable characteristic of DE-LDA is that it can modulate the contribution on the between-class scatter from each class pair through the use of an empirical error function, while preserving the lightweight solvability of LDA. Experiment results seem to demonstrate that DE-LDA yields moderate improvements over LDA on the LVCSR task.
\end{abstract}

Index Terms: speech recognition, feature extraction, linear discriminant analysis, empirical error function

\section{INTRODUCTION}

There are two major reasons why linear discriminant analysis (LDA) has been widely used for speech recognition tasks. First, to reduce the model complexity for lower time and space consumption, LDA can be used to project a higher-dimensional speech feature vector, usually formed by splicing several successive frames for capturing the contextual information of speech signals, into a lower-dimensional space with a minimal loss in discrimination [1]. Second, it has an acceptable characteristic its derivation is simple and fast without needing any iterative optimization technique.

The basic idea behind LDA is to seek a transformation matrix that maximizes the ratio of the between-class scatter of a given data set, which represents the class separability in a geometrical sense [2], to the within-class scatter, which can be also taken as a constraint for metric scaling [3], in a reduced feature space. From the formulation of the above criterion, it appears that LDA does not directly relate itself to classification error rates, the figure of merit that we are interested in most pattern classification tasks. Briefly speaking, the objective of LDA is to maximize class separability in a lower-dimensional feature space, which, however, does not necessarily guarantee better classification accuracy of a given data set. To tackle this problem, Loog integrated a weighting function, which is associated with the theoretical two-class Bayes error rates determined by class-mean differences in a class-pair fashion, into the original between-class scatter statistics [4]. Note that Loog's method is not distribution-free in itself, and furthermore, what it tries to minimize is the upper bound of the global theoretical classification error rate, which is obtained by considering the overall relationships among all classes. Besides, Lee incorporated the empirical classification information from the training data into the derivation of LDA to form a classifier-related objective function [5]. Although Lee's method achieved good recognition results on the test data, the contributions from the empirical classification error rates and the distances between class pairs cannot traded off in an analytical way.

Moreover, LDA can be geometrically viewed as a two-stage procedure [5]. The first stage conducts an orthogonal and whitening transformation of the feature vectors, and the second stage involves a principal component analysis (PCA) on the transformed class means of the feature vectors. Thus, it can be easily seen that the principal axes derived in the second stage will be dominated by large-distance class pairs, and so is the transformation matrix finally derived by LDA. Phrased another way, LDA tends to preserve the class pairs that are already wellseparated, but actually, classes near to each other are more likely to cause confusion and should not be ignored. To alleviate the overemphasis on large-distance class pairs, Li defined a weighting factor to control the contribution made by each class pair on the basis of their Euclidean distance [6]. Naturally, Li's method has also brought about a new question: how moderately should the short-distance class pairs be weighted?

In this paper, by investigating the relationship between the empirical classification error rates of a given ASR and the Mahalanobis distances of the respective class pairs of speech features, we propose a novel reformulation of the LDA criterion, called distance-error coupled LDA (DE-LDA), which can appropriately apply a new weighting function of class-mean differences to solve the aforementioned limitations of LDA. The highlights of DE-LDA are as follows:

1. Inheriting from LDA, DE-LDA is distribution-free and retains the computational simplicity.

2. DE-LDA can be well-conducted on some systems which use not merely Bayesian-based classifiers but other special and complicated ones.

The rest of this paper is organized as follows. Section 2 reviews the classical LDA and some related work. In Section 3, several observations are described and DE-LDA is proposed. Then, the experiment settings and a series of experiments carried out on the Mandarin large vocabulary continuous speech recognition (LVCSR) task are presented in Section 4. Finally, conclusions are drawn in Section 5. 


\section{LDA AND RELATED WORK}

\subsection{LDA}

Let $\mathbf{S}_{B} \in \mathbb{R}^{n \times n}$ and $\mathbf{S}_{W} \in \mathbb{R}^{n \times n}$, respectively, denote the betweenclass and within-class scatter matrices for a data set of $C$ classes and are defined as follows: (Note that $\mathbf{S}_{B}$ can be expressed in terms of class-mean differences [6].)

$$
\begin{aligned}
& \mathbf{S}_{B}=\frac{1}{2} \sum_{i=1}^{c} \sum_{j=1}^{c} p_{i} p_{j}\left(\mathbf{m}_{i}-\mathbf{m}_{j}\right)\left(\mathbf{m}_{i}-\mathbf{m}_{j}\right)^{T}, \\
& \mathbf{S}_{W}=\sum_{i=1}^{c} p_{i} \mathbf{S}_{i} .
\end{aligned}
$$

Here, $\overline{\mathbf{m}}$ denotes the global mean. $p_{i}, \mathbf{m}_{i}$, and $\mathbf{S}_{i}$ denote the prior probability, mean, and covariance matrix of class $i$, respectively. The goal of LDA is to seek a linear transformation $\boldsymbol{\Theta} \in \mathbb{R}^{n \times p}$ that reduces the dimensionality of a given $n$-dimensional feature vector to $p(p<n)$ by maximizing the discrimination criterion [7]:

$$
J_{F}(\boldsymbol{\Theta})=\operatorname{trace}\left(\left(\boldsymbol{\Theta}^{T} \mathbf{S}_{W} \boldsymbol{\Theta}\right)^{-1}\left(\boldsymbol{\Theta}^{T} \mathbf{S}_{B} \boldsymbol{\Theta}\right)\right),
$$

which can be solved as a generalized eigen-analysis problem $\mathbf{S}_{B} \boldsymbol{\theta}_{i}=\lambda_{i} \mathbf{S}_{W} \boldsymbol{\theta}_{i}$ with $\boldsymbol{\theta}_{i}$ and $\lambda_{i}$ being the $i$-th eigenvector and eigenvalue of $\mathbf{S}_{W}^{-1} \mathbf{S}_{B}$.

From the viewpoint of pattern classification, it is worth mentioning that, in the two-class condition, LDA can be implicitly considered to minimize the Bayes classification error by maximizing the Mahalanobis distance between the class pair, while attaining the optimal result only under the assumption that both classes share the same within-class covariance.

\subsection{Weighted LDA}

As mentioned in Section 1, the LDA criterion is not optimal for a multi-class classification task. One of the possible solutions to this problem is to modify the LDA criterion by replacing $\mathbf{S}_{B}$ with the following weighted form:

$\tilde{\mathbf{S}}_{B}=\frac{1}{2} \sum_{i=1}^{c} \sum_{j=1}^{c} p_{i} p_{j} \mathrm{w}\left(\Delta_{i j}\right)\left(\mathbf{m}_{i}-\mathbf{m}_{j}\right)\left(\mathbf{m}_{i}-\mathbf{m}_{j}\right)^{T}$,

where $\mathrm{w}(\bullet)$ is a weighting function of the class-mean difference $\Delta_{i j}$, which is defined by

$\Delta_{i j}=\sqrt{\left(\mathbf{m}_{i}-\mathbf{m}_{j}\right)^{T} \mathbf{S}_{W}^{-1}\left(\mathbf{m}_{i}-\mathbf{m}_{j}\right)}$.

It can be shown that the weighted LDA transformation $\boldsymbol{\Theta}^{\prime}$ can be easily derived by solving the eigen-analysis problem $\tilde{\mathbf{S}}_{B} \boldsymbol{\theta}_{i}^{\prime}=\lambda_{i} \mathbf{S}_{W} \boldsymbol{\theta}_{i}^{\prime}$, which is similar to that of LDA, cf. [5]. Further, note that under the equal-covariance assumption, $\Delta_{i j}$ can be deemed the Mahalanobis distance between classes $i$ and $j$.

In [4], based on the minimization of the theoretical pairwise Bayes errors occurring between any two Gaussian-distributed classes, $\mathrm{w}\left(\Delta_{i j}\right)$ is defined by

$$
\mathrm{W}\left(\Delta_{i j}\right)=\frac{1}{2 \Delta_{i j}^{2}} \operatorname{erf}\left(\frac{\Delta_{i j}}{2 \sqrt{2}}\right),
$$

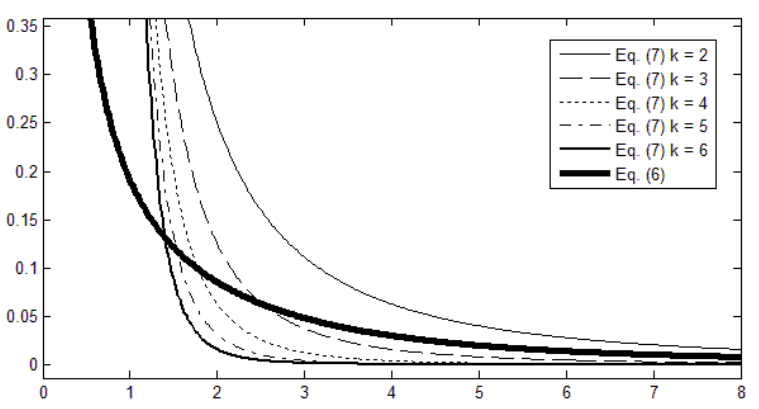

Figure 1: Plot of Eq. (6) and Eq. (7) with various values of $k$. The horizontal axis represents the Mahalanobis distance $\Delta_{i j}$.

where $\operatorname{erf}(\bullet)$ means the error function that is twice the integral of the Gaussian distribution with 0 mean and variance of $1 / 2$. Another weighting function was introduced in [8], which is defined by

$$
\mathrm{W}\left(\Delta_{i j}\right)=\Delta_{i j}^{-k}, k>0 \text {. }
$$

We can see that both of Eqs. (6) and (7) in essence are monotonically decreasing functions of $\Delta_{i j}$ such that those class pairs with large $\Delta_{i j}$ will not be overemphasized (see Fig. 1).

Yet another modification [5], advocating the concept of pairwise class confusion information, takes the practical classification error rates resulted from a given classifier into consideration, rather than operating merely in the Bayesian sense (cf. Eq. (6)). The corresponding weighting function is expressed as

$\mathrm{w}\left(\Delta_{i j}\right)=\alpha+(1-\alpha) \times \mathrm{CI}_{i j}, 0 \leq \alpha \leq 1$,

where $\mathrm{CI}_{i j}$ represents the empirical classification error rate for a sample item that belongs to class $i$ but is misclassified to class $j$, and $\alpha$ denotes an adjustable factor trading off between the empirical classification error rates and the class-mean distances, which can only be set heuristically. We can see that if $\alpha=1$, this approach apparently is reduced to LDA; and if $\alpha=0$, not only will the class pairs with $\mathrm{CI}_{i j} \neq 0$ dominate the whole weightings, but the class pairs with $\mathrm{CI}_{i j}=0$ will get completely ignored.

\section{DISTANCE-ERROR COUPLED LDA}

\subsection{Observations}

We first define the empirical pairwise classification error rate as

$E R_{i j}=\frac{e_{i j}+e_{j i}}{n_{i}+n_{j}}, 1 \leq i<j \leq C$,

where $n_{i}$ denote the number of sample items of phone (or silence) class $i$, and $e_{i j}$ denotes the number of sample items that originally belong to phone (or silence) class $i$ but are misallocated to phone (or silence) class $j$ by the ASR. $E R_{i j}$, in a sense, can be used to measure the confusability between any class pair $i$ and $j$. That is, for class $i$, the higher the value of $E R_{i j}$, the more confusable it would be with class $j$.

Next, in the context of speech recognition, we can divide all of the class pairs into two groups: phone-phone and silence-phone according to their corresponding class labels. For example, if class $i$ belongs to silence, any class pair including class $i$ will be classified into the silence-phone group; on the contrary, the rest 

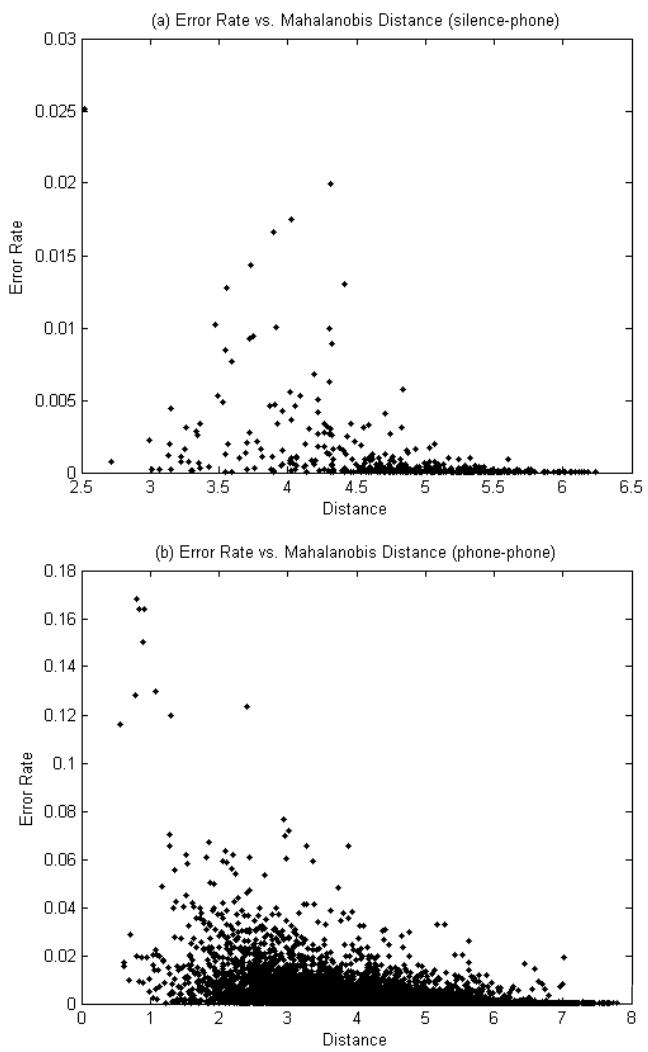

Figure 2: Plots of the empirical pairwise classification error rates versus the corresponding Mahalanobis distances of classmeans pairs for the features of speech training data.

class pairs that do not belong to the silence-phone group will fall into the phone-phone group.

Fig. 2 shows two plots of the empirical pairwise classification error rates versus the corresponding Mahalanobis distances ( $c f$. Eq. (5)) of class-mean pairs for the features of the speech training data used in this paper ( $c f$. Section 4.1), where the Mahalanobis distances are measured in the original feature space, and the empirical pairwise classification error rates (cf. Eq. (9)) are obtained by the LDA-transformed speech features. Note also that dots in Fig. 2(a) represents the class pairs of the silence-phone group, while those in Fig. 2(b) represents the class pairs of the phone-phone group. Several observations can be drawn from these two plots. First, the error rates of most of the class pairs in the silence-phone group are much lower than that in the phone-phone group. Second, the correlation between the two variables, i.e., the distance $\Delta_{i j}$ and the error rate $E R_{i j}$, in the silence-phone group is less pronounced than that in the phone-phone group. Third, in Fig. 2(b), we can roughly depict the relationship between these two variables: class pairs with shorter distances $\left(e . g . \Delta_{i j}<4\right)$ tend to have higher error rates (e.g. $\left.E R_{i j}>0.01\right)$; similarly, class pairs with larger distances (e.g. $\left.\Delta_{i j}>4\right)$ are likely to have lower error rates $\left(\right.$ e.g. $\left.E R_{i j}<0.01\right)$. Such a phenomenon, to some extent, confirms to our expectation: the statistics of class pairs with shorter distances need to be emphasized, while those of the class pairs with larger-distances should be deemphasized instead when deriving the LDA-based feature transformation matrix.

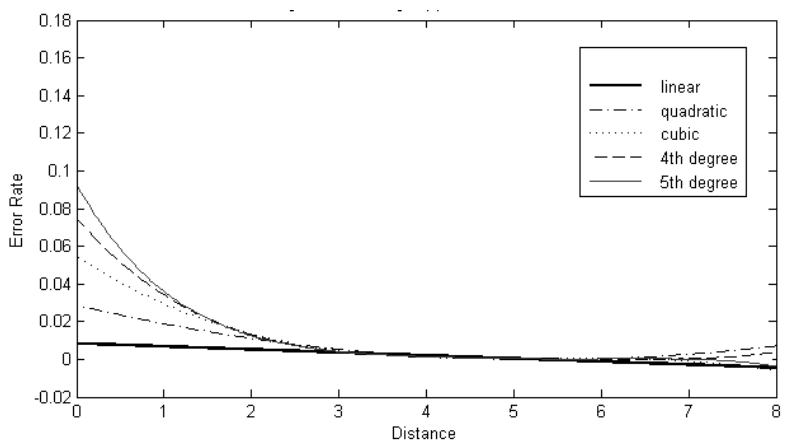

Figure 3: Plot of the polynomial regressions of various degrees based on the data points in Fig. 2(b).

\subsection{The proposed weighting function}

According to the observations made in Section 3.1, it is reasonable to disregard the contributions of the class pairs in the silence-phone group to the LDA derivation, due to their irregularities in the distance-error distribution and less influence on the overall error rates. Henceforth, in this paper we focus merely on the phenomenon revealed by Fig. 2(b) and use the data-fitting (or regression) scheme to find out a function of the Mahalanobis distance, i.e., $\mathrm{E}\left(\Delta_{i j}\right)$, which hopefully can approximate the relationship between the empirical pairwise classification error rate and the corresponding Mahalanobis distance. Data fitting is a mathematical optimization method which, when given a series of data points $\left(u_{i}, v_{i}\right)$ with $i=1, \ldots, n$, attempts to find a function $\mathrm{G}\left(u_{i}\right)$ whose output $\tilde{v}_{i}$ closely approximates $v_{i}$. That is, it minimizes the sum of the squared error (or the squares of the ordinate differences) between the points $\left(u_{i}, \tilde{v}_{i}\right)$ and their corresponding points $\left(u_{i}, v_{i}\right)$ in the data set.

In our work, for example, if $\mathrm{E}\left(\Delta_{i j}\right)$ is supposed to be a quadratic polynomial, i.e., $\mathrm{E}\left(\Delta_{i j}\right)=a \Delta_{i j}^{2}+b \Delta_{i j}+c$, then given all of the data points $\left(\Delta_{i j}, E R_{i j}\right)$ in Fig. 2(b), we can estimate the coefficients of $\mathrm{E}\left(\Delta_{i j}\right), a, b$, and $c$, by minimizing the sum of the squares of $\mathrm{E}\left(\Delta_{i j}\right)-E R_{i j}$ as shown in Eq. (10):

$$
\{\hat{a}, \hat{b}, \hat{c}\}=\arg \min _{a, b, c} \sum_{i=1}^{c-1} \sum_{j=i}^{c}\left[\left(a \Delta_{i j}^{2}+b \Delta_{i j}+c\right)-E R_{i j}\right]^{2} .
$$

The error function $\hat{\mathrm{E}}\left(\Delta_{i j}\right)$ derived by Eq. (10) can be used as our new weighting function:

$$
\mathrm{w}\left(\Delta_{i j}\right)=\hat{\mathrm{E}}\left(\Delta_{i j}\right)=\hat{a} \Delta_{i j}^{2}+\hat{b} \Delta_{i j}+\hat{c} \text {. }
$$

Similar to Eqs. (6) and (7), the curves of the error functions shown in Fig. 3, derived on the basis of data-fitting for polynomials of degree 1 up to degree 5 , are almost monotonically decreasing with the distance. By integrating them respectively into Eq. (4), we not only can relieve the overemphasis problem to some extent, but also can properly couple the realistic pairwise confusion information to the LDA derivation, which is originally based purely on the distance measure of the feature representations.

\section{EXPERIMENTS AND RESULTS}

\subsection{Experiment setup}




\begin{tabular}{|c|c|}
\hline Polynomial Regressions & CER (\%) \\
\hline Linear $\left(1^{\text {st }}\right.$ degree $)$ & 30.76 \\
\hline Quadratic $\left(2^{\text {nd }}\right.$ degree $)$ & 30.90 \\
\hline Cubic $\left(3^{\text {rd }}\right.$ degree $)$ & 30.85 \\
\hline $4^{\text {th }}$ degree & $\mathbf{3 0 . 2 9}$ \\
\hline $5^{\text {th }}$ degree & 30.85 \\
\hline
\end{tabular}

Table 1. The CER results (\%) of DE-LDA, with respect to various degrees of polynomials.

The speech corpus consists of about 200 hours of MATBN Mandarin television news [9]. All the 200 hours of speech data are equipped with corresponding orthographic transcripts, in which about 25 hours of speech data were used to bootstrap the acoustic training. Another set of 1.5 hour speech data of were reserved for testing. On the other hand, the acoustic models chosen here for speech recognition are 112 right-context-dependent INITIAL's and 38 context-independent FINAL's. The acoustic models were trained using the Expectation-Maximization (EM) algorithm.

The recognition lexicon consists of $72 \mathrm{~K}$ words. The language models used in this paper consist of unigram, bigram and trigram models, which were estimated using a text corpus consisting of 170 million Chinese characters collected from Central News Agency (CNA) [10]. The $N$-gram language models were trained using the SRI Language Modeling Toolkit (SRILM).

The speech recognizer was implemented with a left-to-right frame-synchronous Viterbi tree search as well as a lexical prefix tree organization of the lexicon. The recognition hypotheses were organized into a word graph for further language model rescoring [9]. The baseline system with the MFCC features resulted in a character error rate (CER) of $32.16 \%$.

\subsection{Experiment results}

The feature extraction was performed using LDA, DE-LDA, and methods mentioned in Eqs. (6), (7), and (8), respectively, on speech feature vectors consisting of 162 dimensions, which were first spliced by every 9 consecutive 18-dimensional Mel-filterbank feature vectors and then reduced to 39 dimensions. The states of each HMM were taken as the unit for class assignment, and a welltrained HMM-based recognition system was performed to obtain the class alignment of the training utterances. During the speech recognition process we kept track of full state alignment for obtaining the state-level transcriptions of the training data; by comparison with the correct ones, we thus derived the empirical pairwise classification error rates by Eq. (9).

Table 1 shows the results for DE-LDA with polynomial regressions of various degrees, which are derived from the data points in the phone-phone group. As the $4^{\text {th }}$ degree polynomial regression is being used, DE-LDA yields the lowest CER, which has relative improvements of $5.81 \%$ over the system with MFCC baseline and $2.38 \%$ over LDA. The possible reasons why DELDA didn't significantly outperform LDA can be conjectured as follows:

1. Except for the silence-phone group, we didn't get rid of other irregular or insignificant factors, which might affect the generations of the polynomial regressions.

2. We can observe from Fig. 3 that the variations of those polynomials are very slight while $\Delta_{i j}>3$. But in Fig. 2(b),

\begin{tabular}{|c|c|}
\hline Methods & CER (\%) \\
\hline \hline LDA & 31.03 \\
\hline DE-LDA (4 ${ }^{\text {th }}$ degree polynomial) & $\mathbf{3 0 . 2 9}$ \\
\hline Eq. $(6)$ & 30.75 \\
\hline Eq. $(7)(k=5)$ & 30.31 \\
\hline Eq. $(8)(\alpha=0.5)$ & 29.60 \\
\hline
\end{tabular}

Table 2. Comparison among the CER results (\%) of DE-LDA and various LDA-based approaches.

there are still many class pairs with $\Delta_{i j}>3$ but also with higher $E R_{i j}$ that might be deemphasized by the proposed weighting functions.

We also tried to compare the other variants of LDA, as those mentioned in Section 2.2. Table 2 shows that both of the methods conducted based on Eqs. (6) and (7) are comparable to our proposed DE-LDA approach; that is, they also provide moderate performance improvements over LDA as well. The reason might lie in that the tendency of the functions shown in Fig. 1 somewhat fits in with the empirical distance-error distribution shown in Fig. 2(b).

\section{CONCLUSIONS AND FUTURE WORK}

In this paper, we have proposed a new weighting approach called DE-LDA to generalize LDA by modulating the contribution on between-class scatter from each class pair through the use of a class-pair classification error function. The experiment results seem to demonstrate that DE-LDA could successfully capture the relationship between empirical classification error rates and the geometrical distances for better CER performance of the ASR task studied in this paper. Future work includes determining a more appropriate error function than the polynomials and performing DE-LDA in conjunction with a heteroscedastic decorrelation, such as the maximum likelihood linear transform (MLLT) [11].

\section{REFERENCES}

[1] S. Nakagawa and K. Yamamoto, "Evaluation of Segmental Unit Input HMM," in Proc. ICASSP, 1996.

[2] S. S. Wilks, Mathematical Statistics, pp. 577-578, John Wiley \& Sons, New York, 1962.

[3] W. J. Krzanowski, Principles of Multivariate Analysis: A User's Perspective, pp. 298-300, Oxford University Press, New York, 1988.

[4] M. Loog, R.P.W. Duin, and R. Haeb-Umbach, "Multiclass Linear Dimension Reduction by Weighted Pairwise Fisher Criteria," IEEE Trans. on PAMI, vol. 23, no. 7, 2001.

[5] H. S. Lee and B. Chen, "Linear Discriminant Feature Extraction Using Weighted Classification Confusion Information," in Proc. Interspeech, 2008.

[6] Y. Li, Y. Gao, and H. Erdogan, "Weighted Pairwise Scatter to Improve Linear Discriminant Analysis," in Proc. ICSLP, 2000.

[7] K. Fukunaga, Introduction to Statistical Pattern Recognition, pp. 446448, Academic Press, New York, $2^{\text {nd }}$ Ed., 1990.

[8] Y. Liang, C. Li, W. Gong, and Y. Pan, "Uncorrelated Linear Discriminant Analysis Based on Weighted Pairwise Fisher Criterion," Pattern Recognition, vol. 40, no. 12, 2007.

[9] B. Chen, J. W. Kuo, and W. H. Tsai, "Lightly Supervised and DataDriven Approaches to Mandarin Broadcast News Transcription," in Proc. ICASSP, 2004.

[10] H.S. Chiu and B. Chen, "Word Topical Mixture Models for Dynamic Language Model Adaptation," in proc. ICASSP, 2007.

[11] R. A. Gopinath, "Maximum Likelihood Modeling with Gaussian Distributions for Classification," in Proc. ICASSP, 1998. 